\title{
Amino acid transporter inventory of the Selaginella genome
}

\author{
Daniel Wipf $^{1}$ *, Dominique Loqué ${ }^{2}$, Sylvie Lalonde ${ }^{3}$ and Wolf B. Frommer ${ }^{3}$ \\ 1 UMR INRA 1088, CNRS 5184, Université de Bourgogne Plante-Microbe-Environnement, Dijon, France \\ ${ }^{2}$ Joint Bioenergy Institute, Emeryville, CA, USA \\ ${ }^{3}$ Department of Plant Biology, Carnegie Institution for Science, Stanford, CA, USA
}

Edited by:

Angus S. Murphy, Purdue University, USA

Reviewed by:

Yi Ma, University of Connecticut, USA

Joshua Blakeslee, The Ohio State

University, USA

${ }^{*}$ Correspondence:

Daniel Wipf, UMR INRA 1088, CNRS

5184, Université de Bourgogne

Plante-Microbe-Environnement, BP

86510, 21065 Dijon Cedex, France.

e-mail: daniel.wipf@dijon.inra.fr
Amino acids play fundamental roles in a multitude of functions including protein synthesis, hormone metabolism, nerve transmission, cell growth, production of metabolic energy, nucleobase synthesis, nitrogen metabolism, and urea biosynthesis. Selaginella as a member of the lycophytes is part of an ancient lineage of vascular plants that had arisen $\sim 400$ million years ago. In angiosperms, which have attracted most of the attention for nutrient transport so far, we have been able to identify many of the key transporters for nitrogen. Their role is not always fully clear, thus an analysis of Selaginella as a representative of an ancient vascular plant may help shed light on the evolution and function of these diverse transporters. Here we annotated and analyzed the genes encoding putative transporters involved in cellular uptake of amino acids present in the Selaginella genome.

Keywords: Selaginella, amino acid, transporter

\section{INTRODUCTION}

Amino acids play fundamental roles in a multitude of functions including protein synthesis, hormone metabolism, nerve transmission, cell growth, production of metabolic energy, nucleobase synthesis, nitrogen metabolism, and urea biosynthesis. In multicellular organisms, many of the nitrogenous compounds are transported between cells (Lalonde et al., 2004). Long distance transport of organic nitrogen in the plant is probably more complex as compared to sugar transport given the large number of amino acids. The transported substrates are highly diverse, including 20 proteogenic amino acids, GABA, and a variety of amino acid analogs and even oligopeptides, but also many other N-containing compounds. Furthermore, amino acids are not only transported in the phloem but also in xylem, and they exchange between phloem and xylem in a complex manner (Atkins, 2000). If we take into account that nitrogen metabolism is highly compartmentalized; it appears reasonable to expect a large number of transporter genes. Amino acid transporters for cellular import were initially identified by suppression cloning in yeast, and they comprise primarily two major families: the amino acid transporter superfamily 1 (ATF1) and the amino acid-polyamine-choline (APC) transporter superfamily (Frommer et al., 1993, 1995; Lalonde et al., 2004). Transporters for the efflux of amino acids and for transport across the vacuolar membrane may be encoded by uncharacterized members of this family or may be encoded by yet uncharacterized membrane protein genes.

\section{THE AMINO ACID TRANSPORTER SUPERFAMILY 1}

In contrast to the other amino acid transporter families, ATF1 members were first described in plants (Frommer et al., 1993). ATF1 members contain 9-11 putative membrane spanning domains with cytosolic $\mathrm{N}$ - and extracellular $\mathrm{C}$-termini (Chang and Bush, 1997). Within the ATF1 family, six subfamilies were found: amino acid permeases (AAPs), "lysine/histidine" transporters
(LHTs), ProTs, $\gamma$-aminobutyric acid transporters (GATs), ANTs, and AUXs. (i) AAPs have eight members in Arabidopsis. The Arabidopsis AAPs, which mediate $\mathrm{Na}^{+}$-independent, $\mathrm{H}^{+}$-coupled uptake of a wide spectrum of amino acids, represent the bestcharacterized members of the superfamily (Fischer et al., 2002). Slight differences in substrate specificity exist among the different members complicating an exact assessment of their in vivo function. (ii) LHTs were named according to the substrate selectivity originally determined for AtLHT1. However, more detailed studies on the biochemical properties of AtLHT1 and AtLHT2 in Saccharomyces cerevisiae revealed that compared to basic amino acids their affinities for neutral and acidic amino acids are much higher (Hirner et al., 2006). Consequently, the LHTs are now classified as high affinity transporters for these groups of amino acids. LHT1 has been shown to play an important role in the acquisition of amino acids by Arabidopsis roots (Hirner et al., 2006). (iii) ProTs transport the amino acid proline, glycine betaine, and related quaternary ammonium compounds as well as the amino acid $\gamma$-aminobutyric acid (GABA) with moderate or low affinity (Grallath et al., 2005). (iv) GATs transport GABA and GABA-related compounds. (v) Aromatic and neutral amino acid transporter 1 (ANT1)-like proteins, of which only AtANT1 has been characterized so far, functions as a moderate affinity transporter for aromatic and neutral amino acids (Chen et al., 2001). (vi) The AUX (auxin-resistant) subgroup. AtAUX1 was initially reported to be a putative auxin-influx carrier on the basis of genetic data and the analysis of mutants in Arabidopsis. The expression of AtAUX1 in Xenopus oocytes provided unambiguous evidence that AUX1 is capable of importing auxin into cells (Yang et al., 2006).

THE AMINO ACID-POLYAMINE-CHOLINE TRANSPORTER SUPERFAMILY In the absence of structural data, computer-aided predictions of secondary structures were used to categorize plant APC 
Table 1 | Number of gene from the different amino acid transporters subfamilies found in Arabidopsis, Rice, Selaginella, Physcomitrella, and Chlamydomonas genomes (nd, not determined).

\begin{tabular}{|c|c|c|c|c|c|c|c|}
\hline \multirow[t]{2}{*}{ Gene function } & \multirow[t]{2}{*}{ Gene name } & \multirow{2}{*}{$\begin{array}{l}\text { Gene used } \\
\text { for query }\end{array}$} & \multicolumn{5}{|c|}{ Number of putative orthologs } \\
\hline & & & Arabidopsis & Rice & Selaginella & Physcomitrella & Chlamydomonas \\
\hline \multirow[t]{4}{*}{ Amino acid transport } & AAP, LHT, ProT, Aux, GAT, ANT1 & & 48 & 57 & nd & nd & $\mathrm{Nd}$ \\
\hline & LAT & AtLAT1 & 5 & 4 & 4 & 3 & 0 \\
\hline & CAT & AtCAT1 & 10 & 8 & 5 & 6 & 3 \\
\hline & DIT2 & AtDIT2 & 2 & 2 & 3 & 4 & 2 \\
\hline
\end{tabular}

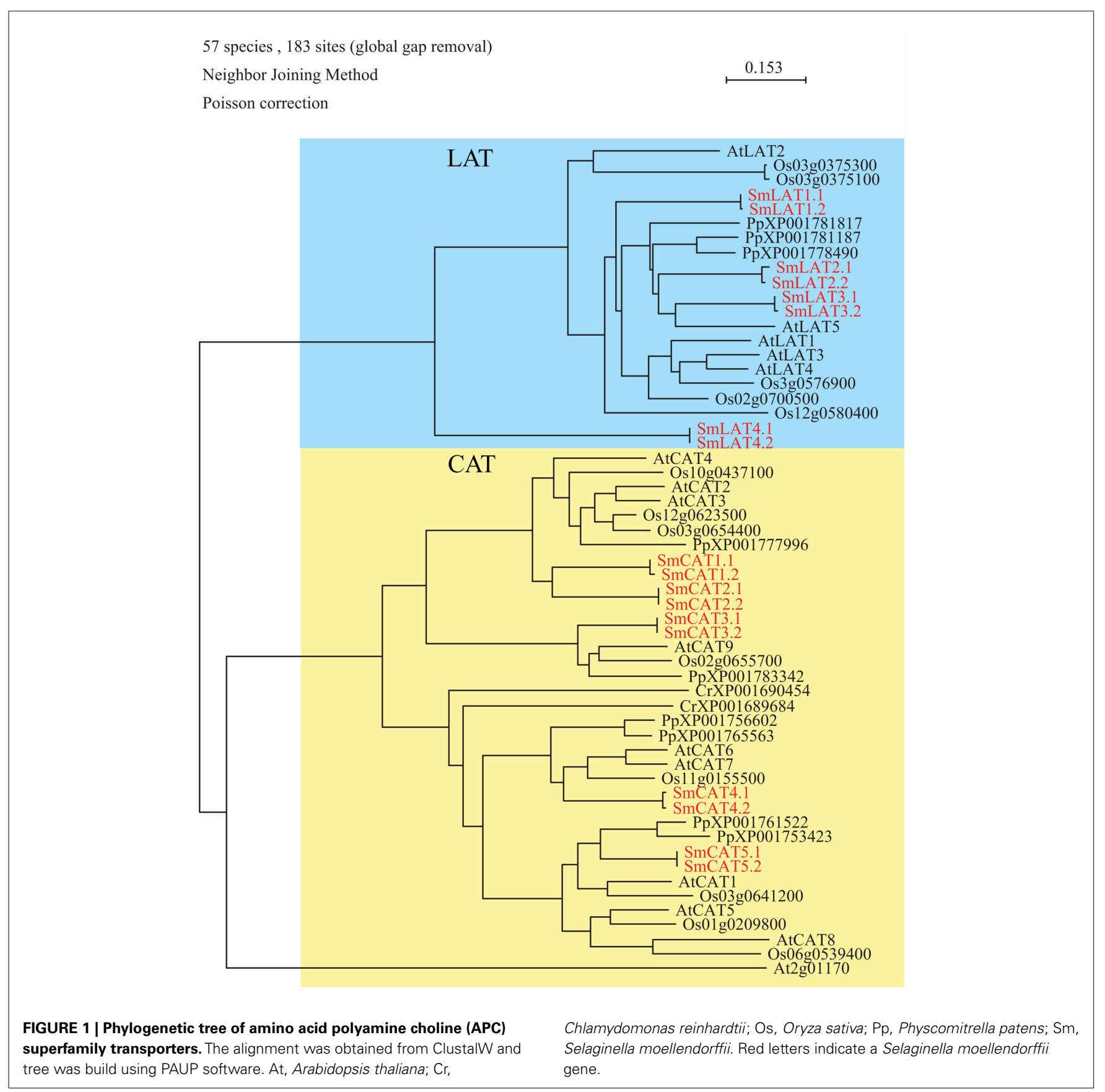




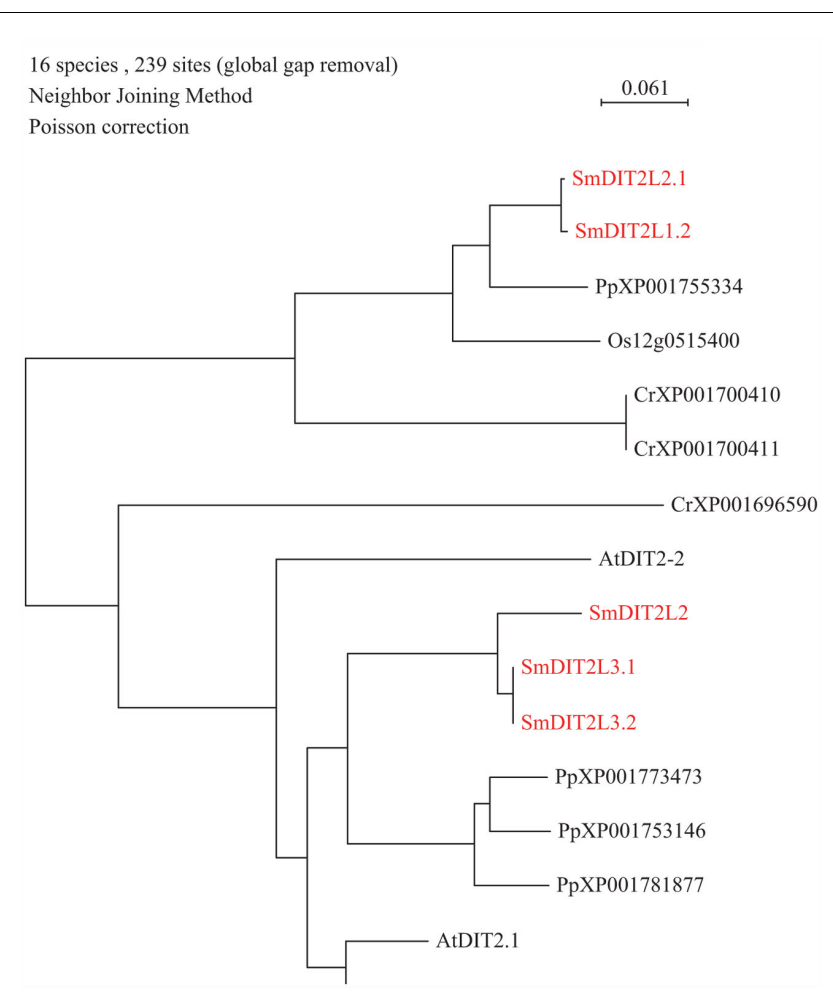

FIGURE 2 | Phylogenetic tree of DiT2 (dicarboxylate) transporter family. The alignment was obtained from ClustalW and tree was build using PAUP software. At, Arabidopsis thaliana; $\mathrm{Cr}$, Chlamydomonas reinhardtii; Os, Oryza sativa; Pp, Physcomitrella patens; Sm, Selaginella moellendorffii. Red letters indicate a Selaginella moellendorffii gene.

transporters into two subgroups (http://aramemnon.botanik. uni-koeln.de/). Members of the cationic amino acid transporters (CATs) are probably built from 14 putative transmembrane domains, with homologs found in animals (Wipf et al., 2002). The second subfamily comprises proteins with 12 putative transmembrane domains (Wipf et al., 2002). In short, amino acid transport mediated by members of the APC family from different organisms is diverse, uses $\mathrm{Na}^{+}$- or $\mathrm{H}^{+}$-coupling by co- or antiport and serve functions in uptake and nutrition (Su et al., 2004; Yang et al., 2010).

So far, the amino acid transporters that have been identified fall into at least five gene families (Rentsch et al., 2007) and display different substrate selectivity and affinity as well as distinct subcellular localization (Wipf et al., 2002). Recently at bidirectional amino acid transporter 1 (AtBAT1) a new AA transporter, which is not a member of any previously defined amino acid transporter family, was identified (Dundar and Bush, 2009). Direct measurement of amino acid transport and yeast growth experiments demonstrated that AtBAT1 exhibits transport activity for alanine, arginine, glutamate, and lysine, but not for GABA or proline. Significantly, unlike other amino acid transporters described in plants to date, AtBAT1 displayed both export and import activity. BAT1 is a single copy gene in the Arabidopsis genome, and its mRNA is ubiquitously expressed in all organs. BAT1 may thus be key for cellular efflux, a step needed for transport between cells and organs, similar as the sucrose efflux transporters of the SWEET family (Chen et al., 2011).

Selaginella is part of an ancient lineage of vascular plants that had arisen $\sim 400$ million years ago. An analysis of Selaginella as a representative of an ancient vascular plant may help shed light on the evolution and function of the diverse nutrient transporters. Here we analyzed the genes for transporters involved in cellular uptake of amino acids present in the Selaginella genome. We carried out a similar analysis for the Selaginella complement of genes for transporters involved in cellular uptake of ammonium, urea, and cellular uptake of sugars (DeMichele et al., FiPS, in revision; Lalonde et al., FiPS, in revision).

\section{MATERIALS AND METHODS}

The Selaginella moellendorffii genome was annotated using the JGI Annotation pipeline, which combines several gene prediction, annotation, and analysis tools. Gene predictors used for annotation include Fgenesh, Fgensh+, and Genewis (Banks et al., 2011). Over 110,000 Selaginella ESTs were clustered, converted into putative full-length (FL) genes and directly mapped to genomic sequences or used to extend predicted gene models into FL genes by adding $5^{\prime}$ and/or $3^{\prime}$ UTRs to the models. Since multiple gene models were generated for each locus, a single representative model was chosen based on homology and EST support and used for further analysis.

All predicted gene models were functionally annotated by homology to annotated genes in the NCBI non-redundant set. Each gene model was analyzed for domain content and structure using InterProScan and classified according to Gene Ontology, eukaryotic orthologous groups (KOGs) and KEGG metabolic pathways. In total, 34,292 gene models were predicted for the Selaginella assembly (Banks et al., 2011).

Gene predictions were checked by aligning Selaginella amino acid transporter sequences with known plant amino acid transporters in the ClustalW software (Thompson et al., 1994). Phylogenetic trees were build using PAUP software (Swofford, 1998).

\section{RESULTS AND DISCUSSION AMINO ACID TRANSPORT IN SELAGINELLA}

Analysis of the $S$. moellendorffii genome indicated the presence of nine amino acid transporters belonging to the APC transporter superfamily (Table 1; Figure 1), four belonging to the L-type Amino acid Transporter clade (respectively annotated as LAT1.1 (and a putative allele LAT1.2), LAT2.1 (and a putative allele LAT2.2), LAT3.1 (and a putative allele LAT3.2), and LAT4.1 (and a putative allele LAT4.2), and five belonging to the CAT clade (respectively annotated as CAT1.1 (and a putative allele CAT1.2), CAT2.1 (and a putative allele CAT2.2), CAT3.1 (and a putative allele CAT3.2), CAT 4.1 (and a putative allele CAT4.2), and CAT5.1 (and a putative allele CAT5.2). Thus Selaginella possesses the same number of LATs as rice (4), which is similar to the number found in Arabidopsis (5) (Table 1). In Physcomitrella patens only three LAT homologs were found whereas none were found in the Chlamydomonas reinhardtii genome. According to our analyses, and to be able to perform a direct comparison, the annotation in Physcomitrella needs to be improved, since in many cases the start codon does not appear to be assigned correctly, 
37 species , 193 sites (global gap removal)

Neighbor Joining Method

$\stackrel{0.116}{\longmapsto}$

Poisson correction

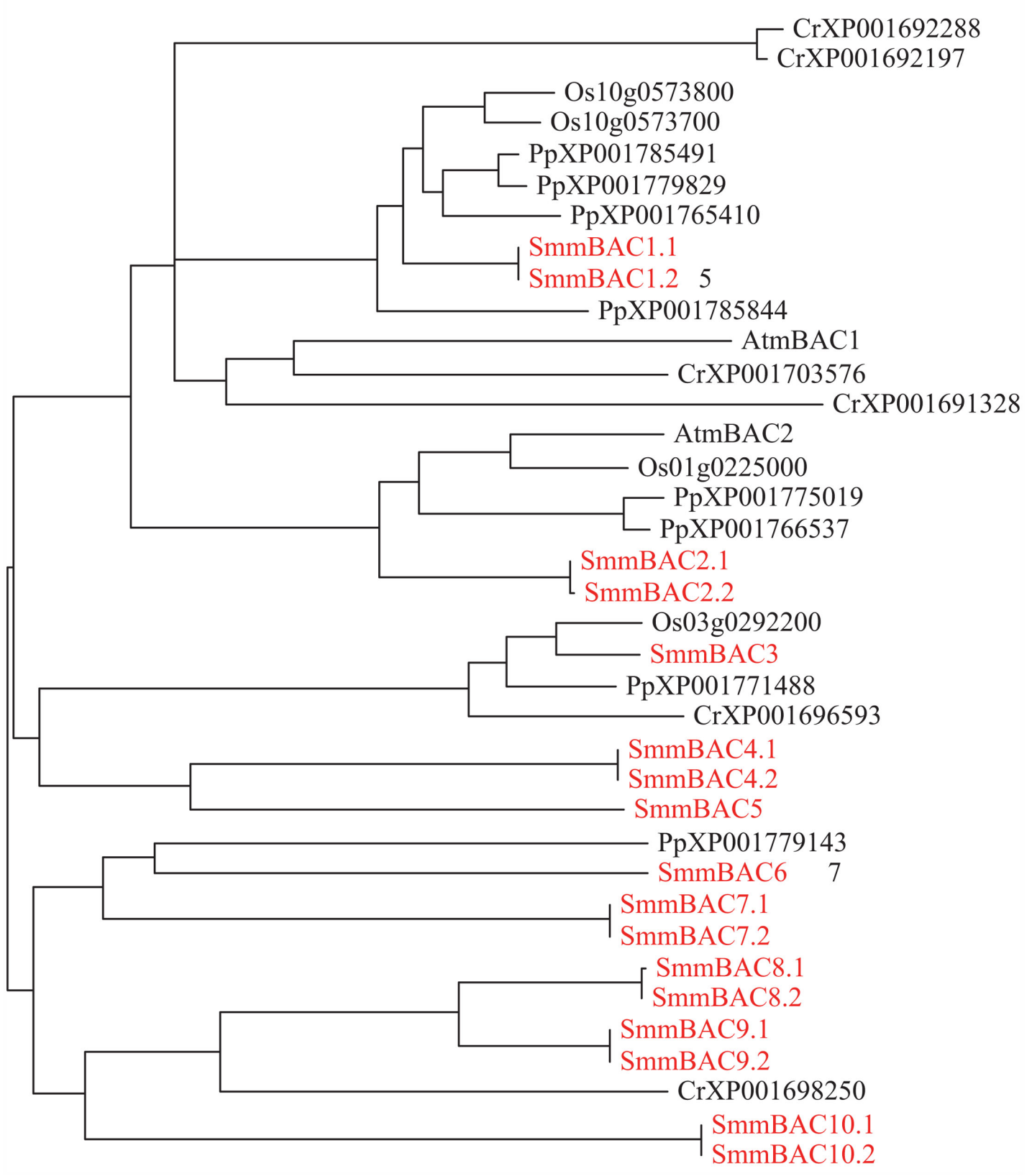

FIGURE 3 | Phylogenetic tree of mitochondrial basic amino acid carriers (mBAC) transporter family. The alignment was obtained from ClustalW and tree was build using PAUP software. At, Arabidopsis thaliana; $\mathrm{Cr}$,
Chlamydomonas reinhardtii; Os, Oryza sativa; Pp, Physcomitrella patens; Sm, Selaginella moellendorffii. Red letters indicate a Selaginella moellendorffii gene. putative sequencing errors may have led to apparent frame shifts, and in several cases intron locations are possibly not assigned correctly. The Selaginella annotation carried out here and the gene models may prove useful for improving the Physcomitrella genome annotation.
Concerning the second clade of amino acid transporters from the APC superfamily the situation is different (Figure 1). Less CAT type transporters were found in mosses and green algae (Selaginella: 5, Physcomitrella: 6, Chlamydomonas: 3) compared to 8 in rice, and 10 in Arabidopsis (Table 1). 


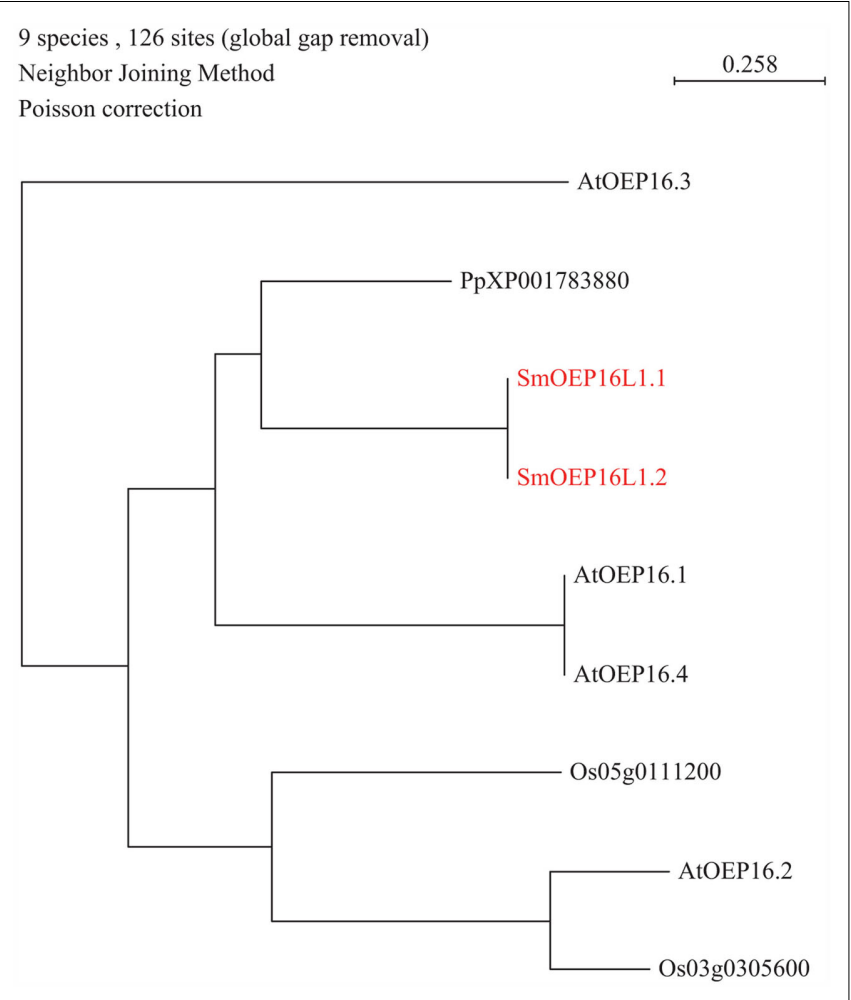

FIGURE 4 | Phylogenetic tree of outer envelope plastid (OEP) transporter family. The alignment was obtained from ClustalW and tree was build using PAUP software. At, Arabidopsis thaliana; Os, Oryza sativa; Pp, Physcomitrella patens; Sm, Selaginella moellendorffii. Red letters indicate a Selaginella moellendorffii gene.

\section{AMINO ACID TRANSPORTERS IN OTHER GENE FAMILIES}

Several other protein families appear to be responsible for transport across the membranes of organelles. Transporters have been identified that facilitate amino acid transport from the cytosol into plastids. In the outer envelope, OEP16 from pea forms a cationselective high-conductance channel with a strong bias for amino acids and amines (Pohlmeyer et al., 1997). So far, the only amino acid transporter identified to operate at the inner plastid envelope is DiT2.1 (dicarboxylate transport), belonging to the divalent anion: $\mathrm{Na}^{+}$symporter (DASS) family. DiT2.1 functions in glutamate/malate exchange essential for the photorespiration (Renné et al., 2003).

Mitochondrial amino acid transporters have been found in plants that, like the yeast and animal mitochondrial transport systems, are members of the mitochondrial carrier family (MCF). In Arabidopsis, two AtmBACs (mitochondrial basic amino acid carriers) have been functionally characterized, and their substrate selectivity resembles that of the corresponding human proteins (Catoni et al., 2003).

\section{AtDIT2 homologs}

So far, the only amino acid transporter identified to operate at the inner plastid envelope is AtDiT2.1 (dicarboxylate transport), belonging to the DASS family. DiT2.1 functions in glutamate/malate exchange essential in the photorespiratory pathway; transport activity for its closest homolog, AtDiT2.2, has not been demonstrated yet. Analysis of S. moellendorffii genome indicates the presence of three putative DIT2 amino acid transporters (DIT2L2.1, DIT2L2.2, and DIT2L3.1 (putative paralog DIT2L3.2; Table 1; Figure 2). DIT2L2 is most closely related to DIT2L3.1 and 3.2. It is interesting to note that a similar numbers of DIT2s is found in mosses and algae (Selaginella: 3, Physcomitrella: 4, Chlamydomonas: 2) as in both Arabidopsis (2) and rice (3) (Table 1).

\section{AtmBAC1 homologs}

Mitochondrial amino acid transporters have been found in plants that, as in yeast and animals, are members of the MCF. In Arabidopsis, two AtmBACs (mitochondrial basic amino acid carriers) have been functionally characterized. Their substrate selectivity resembles that of the corresponding human proteins. Up to 10 genes homologous to AtmBAC1 were found in S. moellendorffii (Table 1; Figure 3). At present, it is not possible to determine which of these 10 members of the MCF may transport amino acids, because MCFs transport a wide spectrum of compounds.

\section{AtOEP16.1 homologs}

A major part of amino acid assimilation and biosynthesis of plants occurs in plastids. Amino acids pass the outer envelope via OEP proteins forming cation-selective high-conductance channels permeable to amines and amino acids. A single OEP16 homolog was found in both the S. moellendorffii and P. patens genomes (Table 1; Figure 4), whereas no homolog was found in the C. reinhardtii genome. Notably, part of the nitrogen assimilation pathway is cytosolic in algae (Fischer and Klein, 1988). It will be interesting to study amino acid transport across the plastid membrane in green algae to determine which proteins may be responsible for transport of amino acids across the outer envelope. Mosses and higher plants may have shifted nitrogen assimilation to the plastid and may thus have required high capacity transporters such as OEP16 to sustain high flux rates.

\section{CONCLUSION}

Taken together, the haploid genome of the spikemoss Selaginella contains at least 23 amino acid transporter homologs. In addition, Selaginella also contains a single homolog of the plastid amino acid transporters (AtOEP16.1 homologs) whereas Chlamydomonas contains none, which could reflect evolutionary differences in the physiology of amino acid metabolism and transport from green algae to mosses to higher plants. Unfortunately, at present it is not possible to assess the physiological function of these transporters in Selaginella due to the lack of tools for gene replacement and transformation. However the genome of the moss Physcomitrella appears to contain a similar complement of amino acid transporters, and all necessary tools for analyzing the physiological function of these genes are available. The identification of the amino acid transporters from the "ancient" Selaginella and the understanding of their functions may contribute to our understanding of the evolution of nitrogen compartmentation and translocation. 


\section{ACKNOWLEDGMENTS}

The authors are grateful to $\mathrm{M}$. Avolio for critical reading of the manuscript. The work reported in this review article was supported by the Burgundy Regional Council and the Agence
National de la Recherche (project TRANSMUT, ANR-10-BLAN1604-01) to Daniel Wipf, as well as from the Department of Energy (DE-FG02-04ER15542) and The National Science Foundation (MCB-1021677) to Wolf B. Frommer.

\section{REFERENCES}

Atkins, C. (2000). Biochemical aspects of assimilate transfers along the phloem path: N-solutes in lupins. Aust. J. Plant Physiol. 27, 531-537.

Banks, J. A., Nishiyama, T., Hasebe, M., Bowman, J. L., Gribskov, M., Depamphilis, C., Albert, V. A., Aono, N., Aoyama, T., Ambrose, B. A., Ashton, N. W., Axtell, M. J., Barker, E., Barker, M. S., Bennetzen, J. L., Bonawitz, N. D., Chapple, C., Cheng, C., Correa, L. G. G., Dacre, M., Debarry, J., Dreyer, I., Elias, M., Engstrom, E. M., Estelle, M., Feng, L., Finet, C. D., Floyd, S. K., Frommer, W. B., Fujita, T., Gramzow, L., Gutensohn, M., Harholt, J., Hattori, M., Heyl, A., Hirai, T., Hiwatashi, Y., Ishikawa, M., Iwata, M., Karol, K. G., Koehler, B., Kolukisaoglu, U., Kubo, M., Kurata, T., Lalonde, S., Li, K., Li, Y., Litt, A., Lyons, E., Manning, G., Maruyama, T., Michael, T. P., Mikami, K., Miyazaki, S., Morinaga, S. Ä., Murata, T., Mueller, Äêroeber, B., Nelson, D. R., Obara, M. Oguri, Y., Olmstead, R. G., Onodera, N., Petersen, B. L., Pils, B., Prigge, M., Rensing, S. A., Riaño-Pachón, D. M., Roberts, A. W., Sato, Y., Scheller, H. V., Schulz, B., Schulz, C., Shakirov, E. V., Shibagaki, N., Shinohara, N., Shippen, D. E., Sørensen, I., Sotooka, R., Sugimoto, N., Sugita, M., Sumikawa, N., Tanurdzic, M. Theissen, G., Ulvskov, P., Wakazuki, S., Weng, J. K., Willats, W. W., Wipf, D., Wolf, P. G., Yang, L., Zimmer, A. D., Zhu, Q., Mitros, T., Hellsten, U., Loqué, D., Otillar, R., Salamov, A., Schmutz, J., Shapiro, H., Lindquist, E., Lucas, S., Rokhsar, D., and Grigoriev, I. V. (2011). The Selaginella genome identifies genetic changes associated with the evolution of vascular plants. Science 332, 960-963.

Catoni, E., Desimone, M., Hilpert, M., Wipf, D., Kunze, R., Schneider, A., Flügge, U. I., Schumacher, K., and Frommer, W. B. (2003).
Expression pattern of a nuclear encoded mitochondrial arginineornithine translocator gene from Arabidopsis. BMC Plant Biol. 3, 1. doi:10.1186/1471-2229-3-1

Chang, H.-C., and Bush, D. R. (1997). Topology of NAT2, a prototypical example of a new family of amino acid transporters. J. Biol. Chem. 272, 30552-30557.

Chen, L. Q., Qu, X. Q., Hou, B. H., Osorio, S., Fernie, A. R., and Frommer, W. B. (2011). Cellular sucrose efflux as a key step in phloem loading. Science 335, 207-211.

Chen, L. S., Ortiz-Lopez, A., Jung, A., and Bush, D. R. (2001). ANT1, an aromatic and neutral amino acid transporter in Arabidopsis. Plant Physiol. 125, 1813-1820.

Dundar, E., and Bush, D. R. (2009). BAT1, a bidirectional amino acid transporter in Arabidopsis. Planta 229, 1047-1056.

Fischer, P., and Klein, U. (1988). Localization of nitrogen-assimilating enzymes in the chloroplast of Chlamydomonas reinhardtii. Plant Physiol. 88, 947-952.

Fischer, W. N., Loo, D. D. F., Koch, W., Ludewig, U., Boorer, K. J., Tegeder, M., Rentsch, D., Wright, E. M., and Frommer, W. B. (2002). Low and high affinity amino acid $\mathrm{H}^{+}$. cotransporters for cellular import of neutral and charged amino acids. Plant J. 29, 717-731.

Frommer, W. B., Hummel, S., and Riesmeier, J. W. (1993). Yeast expression cloning of a cDNA encoding a broad specificity amino acid permease from Arabidopsis thaliana. Proc. Natl. Acad. Sci. U.S.A. 90, 5944-5948.

Frommer, W. B., Hummel, S., Unseld, M., and Ninnemann, O. (1995). Seed and vascular expression of a high affinity transporter for cationic amino acids in Arabidopsis. Proc. Natl. Acad. Sci. U.S.A. 92, 12036-12040.
Grallath, S., Weimar, T., Meyer, A., Gumy, C., Suter-Grotemeyer, M., Neuhaus, J. M., and Rentsch, D. (2005). The AtProT family. Compatible solute transporters with similar substrate specificity but differential expression patterns. Plant Physiol. 137, 117-126.

Hirner, A., Ladwig, F., Stransky, H., Okumoto, S., Keinath, M., Harms, A., Frommer, W. B., and Koch, W. (2006). Arabidopsis LHT1 is a high-affinity transporter for cellular amino acid uptake in both root epidermis and leaf mesophyll. Plant Cell 18, 1931-1946.

Lalonde, S., Wipf, D., and Frommer, W. B. (2004). Transport mechanisms for carbon and nitrogen between source and sink. Annu. Rev. Plant Biol. 55, 341-372.

Pohlmeyer, K., Soll, J., Steinkamp, T., Hinnah, S., and Wagner, R. (1997). Isolation and characterization of an amino acid-selective channel protein present in the chloroplastic outer envelope membrane. Proc. Natl. Acad. Sci. U.S.A. 94 9504-9509.

Renné, P., Dressen, U., Hebbeker, U. Hille, D., Flügge, U. I., Westhoff, P., and Weber, A. P. M. (2003). The Arabidopsis mutant dct is deficient in the plastidic glutamate/malate translocator DiT2. Plant J. 35, 316-331.

Rentsch, D., Schmidt, S., and Tegeder, M. (2007). Transporters for uptake and allocation of organic nitrogen compounds in plants. FEBS Lett. 581, 2281-2289.

Su, Y. H., Frommer, W. B., and Ludewig, U. (2004). Molecular and functional characterization of a family of amino acid transporters from Arabidopsis. Plant Physiol. 136, 3104-3113.

Swofford, D. L. (1998). PAUP*. Phy logenetic Analysis Using Parsimony (*and Other Methods). Version 4. Sunderland, MA: Sinauer Associates.

Thompson, J. D., Higgins, D. G., and Gibson, T. J. (1994). CLUSTAL W: improving the sensitivity of progressive multiple sequence alignment through sequence weighting, position-specific gap penalties and weight matrix choice. Nucleic Acids Res. 22, 4673-4680.

Wipf, D., Ludewig, U., Tegeder, M., Rentsch, D., Koch, W., and Frommer, W. B. (2002). Conservation of amino acid transporters in fungi, plants and animals. Trends Biochem. Sci. 27, 139-147.

Yang, H. Y., Bogner, M., Stierhof, Y. D., and Ludewig, U. (2010). $\mathrm{H}^{+}$. independent glutamine transport in plant root tips. PLoS ONE 5, e8917. doi:10.1371/journal.pone.0008917

Yang, Y. D., Hammes, U. Z., Taylor, C. G., Schachtman, D. P., and Nielsen, E. (2006). High-affinity auxin transport by the AUX1 influx carrier protein. Curr. Biol. 16, 1123-1127.

Conflict of Interest Statement: The authors declare that the research was conducted in the absence of any commercial or financial relationships that could be construed as a potential conflict of interest.

Received: 02 November 2011; accepted: 10 February 2012; published online: 09 April 2012.

Citation: Wipf D, Loqué D, Lalonde S and Frommer WB (2012) Amino acid transporter inventory of the Selaginella genome. Front. Plant Sci. 3:36. doi: 10.3389/fpls.2012.00036

This article was submitted to Frontiers in Plant Evolution and Development, a specialty of Frontiers in Plant Science. Copyright (C) 2012 Wipf, Loqué, Lalonde and Frommer. This is an open-access article distributed under the terms of the Creative Commons Attribution Non Commercial License, which permits noncommercial use, distribution, and reproduction in other forums, provided the original authors and source are credited. 